\title{
Electrical Modulation of Fano Resonance in Plasmonic Nanostructures Using Graphene
}

\author{
Naresh K. Emani, ${ }^{\dagger, \S}$ Ting-Fung Chung, ${ }^{\ddagger}, \S$ Alexander V. Kildishev, ${ }^{\dagger, \S}$ Vladimir M. Shalaev, ${ }^{\dagger, \S}$
}

Yong P. Chen, $\dagger, \ddagger, \S$ and Alexandra Boltasseva ${ }^{* \dagger, \|, \S}$

${ }^{\dagger}$ School of Electrical and Computer Engineering, ${ }^{\star}$ Department of Physics, and ${ }^{\S}$ Birck Nanotechnology Center, Purdue University, West Lafayette, Indiana 47907, United States

"DTU Fotonik, Department of Photonics Engineering, Technical University of Denmark, Lyngby, DK-2800, Denmark

\section{Supporting Information}

ABSTRACT: Pauli blocking of interband transistions gives rise to tunable optical properties in single layer graphene (SLG). This effect is exploited in a graphene-nanoantenna hybrid device where Fano resonant plasmonic nanostructures are fabricated on top of a graphene sheet. The use of Fano resonant elements enhances the interaction of incident radiation with the graphene sheet and enables efficient electrical modulation of the plasmonic resonance. We observe electrically controlled damping in the Fano resonances occurring at approximately $2 \mu \mathrm{m}$, and the results are verified

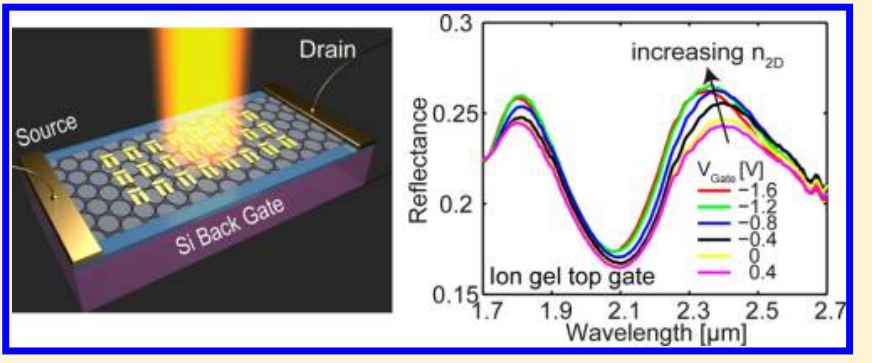
by full-wave 3D finite-element simulations. Our approach can be used for development of next generation of tunable plasmonic and hybrid nanophotonic devices.

KEYWORDS: Graphene, Fano resonance, plasmonics, tunable resonances

$\mathrm{P}$ lasmonics ${ }^{1-3}$ enables unique nanoscale optical devices with novel functionalities. Potential applications include information processing, ${ }^{4}$ localized heating for magnetic recording $^{5}$ and photothermal therapy ${ }^{6}$ to single molecule sensing. ${ }^{7}$ The ability to dynamically modulate the plasmon resonance offers several advantages such as the ability to adjust the spectral window of the operation, multiarray biosensing ${ }^{8}$ and improving the sensitivity of detection by improving signal-tonoise ratio. ${ }^{7}$ Indeed a range of methods including electrical, ${ }^{9}$ optical, ${ }^{10}$ mechanical, ${ }^{11,12}$ and liquid crystal ${ }^{13}$ approaches have been used to tune metamaterials. More recently, alternative plasmonic materials such as transparent conducting oxides (indium tin oxide (ITO), Al- and Ga-doped zinc oxide (AZO and GZO) and others) have been highlighted for tunable plasmonic and metamaterial applications. ${ }^{14,15}$ The optical properties of noble metals, which have long been used as materials of choice due to their large carrier concentrations $\left(10^{22} \mathrm{~cm}^{-3}\right)$ required to support surface plasmon oscillations, ${ }^{16}$ are hard to tune. ${ }^{15}$ In contrast, doped semiconductors, which offer slightly lower carrier concentrations $\left(10^{20} \mathrm{~cm}^{-3}\right)$, are easier to tune. In this context it is not surprising that graphene, ${ }^{17,18}$ a $2 \mathrm{D}$ semimetal with a linear dispersion, exhibits highly tunable optical properties. Tunable surface plasmons in graphene have already been demonstrated in the mid-IR ${ }^{19-22}$ and far- $\mathrm{IR}^{23-25}$ spectra. Graphene has also been used in a variety of optical devices like split-ring resonators, ${ }^{26}$ optical modulators, ${ }^{27}$ and photodetectors ${ }^{28,29}$ and has been proposed as a platform for optical devices. ${ }^{30,31}$ We recently demonstrated electrically controlled damping of the plasmon resonance in metal bowtie antennas placed on top of a graphene layer at the mid-IR wavelengths. ${ }^{32}$ Subsequently, there were a number of devices that demonstrate the tuning of plasmonic antennas ${ }^{33-36}$ and photonic crystal cavities ${ }^{37,38}$ using graphene. However, strong electrical tunability of plasmonic resonances using graphene has so far been experimentally demonstrated only at the mid-IR wavelegnths. Tunable devices at the technologically important visible and/or near-IR wavelengths (for example, for various biosensing applications and telecommunications) have not been realized so far. In this Letter, we show that graphene can be used to effectively modulate the Fano resonance in metal nanostructures. The achieved tunability is much stronger than in our previous work ${ }^{32}$ and the wavelength of operation is closer to the near-IR wavelength range where many potential applications exist.

The Fano resonance results from the interference of a narrow resonance with a broad continuum of states leading to enhanced transmission and reduced reflection, identifiable by the characteristic Fano line shape. ${ }^{39}$ Fano resonance using metal nanostructures has been reported in dolmen, ${ }^{40}$ nonconcentric ring/disk cavities and oligomer ${ }^{41}$ geometries, and has been widely investigated because of its large sensitivity to the local environment. ${ }^{39}$ In our experiments, we first fabricated a graphene field effect transistor (FET) by transfer of CVD

Received: August 30, 2013

Revised: November 27, 2013

Published: December 4, 2013 
grown single layer graphene (SLG) onto a highly p-doped $\mathrm{Si}$ / $\mathrm{SiO}_{2}(300 \mathrm{~nm})$ substrate. ${ }^{42,43}$ Thereafter, we fabricated the Fano resonant dolmen structures on top of the SLG as shown in Figure 1a. This enables us to exploit the large sensitivity of

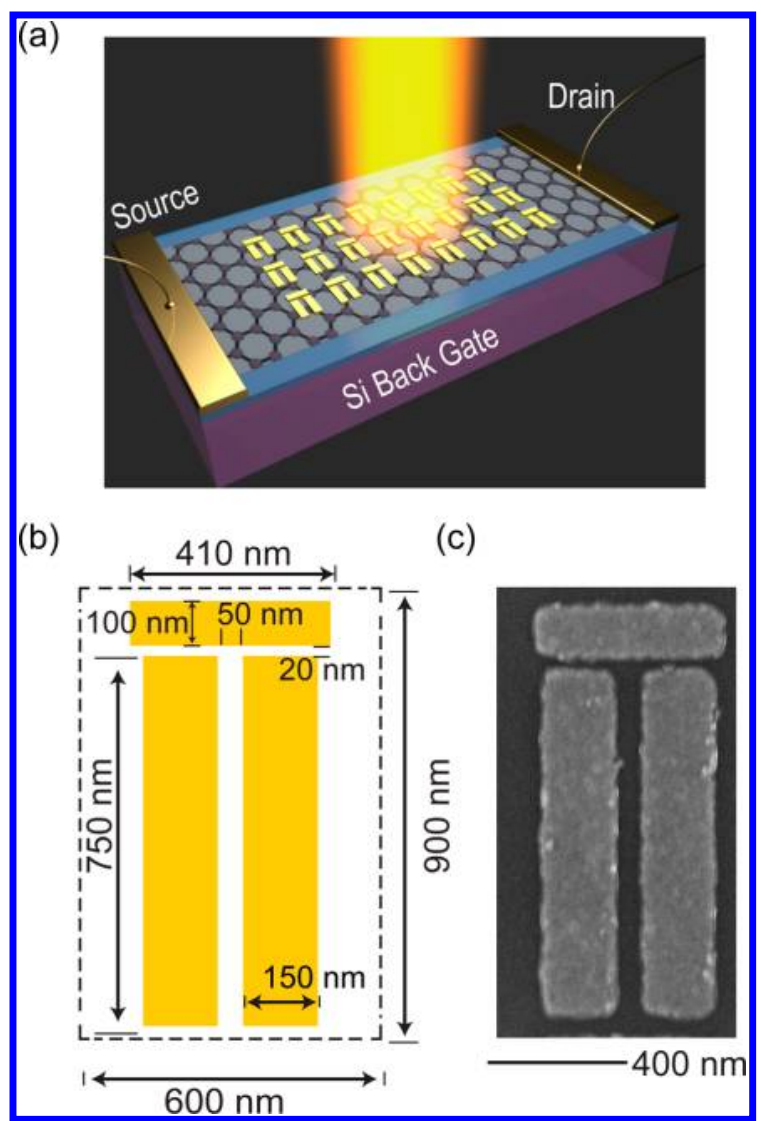

Figure 1. (a) Schematic illustrating the Fano resonant plasmonic antennas fabricated on top of SLG used in our experiments. The optical measurements were performed using an FTIR spectrometer with an attached microscope in reflection mode. (b) Geometry of the dolmen structure used in our experiment. When the incident light is polarized along the horizontal rod it excites a dipolar (bright) mode, and simultaneously a quadrupolar(dark) mode is excited in the vertical rod pair. The dimensions in the structure were optimized to achieve overlapping of these modes so that a Fano resonance dip around 1.9 $\mu \mathrm{m}$. (c) Scanning electron micrograph showing the sample fabricated using e-beam lithography. The scale bar is shown below the image. Doses in the lithography were optimized to achieve reliable gaps of 20 and $50 \mathrm{~nm}$ required by the design.

the resonance to local environment and also achieve electrical control. The optical properties of graphene depend strongly on the carrier density in the graphene sheet. At the charge neutral point, interband transitions are allowed at any wavelength and contribute to the characteristic $2.3 \%$ absorption in graphene. However, when the graphene sheet is doped, by means of applying a back gate voltage as shown in Figure la or using a ionic liquid as a top gate, some of these interband transitions are blocked and the absorption of graphene exhibits step-like behavior around the interband threshold given by the Fermi energy $\left(2 E_{\mathrm{F}}\right){ }^{44,45}$ The optical properties of graphene are captured very well using the random phase approximation $(\mathrm{RPA})^{44}$ and is described by the optical conductivity as below

$$
\begin{aligned}
\sigma(\omega)= & \frac{2 e^{2} \omega_{\mathrm{T}}}{\pi \hbar} \frac{i}{\omega+i \tau^{-1}} \log \left[2 \cosh \left(\frac{\omega_{\mathrm{F}}}{2 \omega_{\mathrm{T}}}\right)\right] \\
& +\frac{e^{2}}{4 \hbar}\left[H\left(\frac{\omega}{2}\right)+i \frac{2 \omega}{\pi} \int_{0}^{\infty} \frac{H\left(\frac{\omega^{\prime}}{2}\right)-H\left(\frac{\omega}{2}\right)}{\omega^{2}-\omega^{\prime 2}} \mathrm{~d} \omega^{\prime}\right]
\end{aligned}
$$

where $H(\omega)=\sinh \left(\omega / \omega_{\mathrm{T}}\right) /\left[\cosh \left(\omega_{\mathrm{F}} / \omega_{\mathrm{T}}\right)+\cosh \left(\omega / \omega_{\mathrm{T}}\right)\right]$, $\omega_{\mathrm{F}}=E_{\mathrm{F}} / \hbar, \omega_{\mathrm{T}}=k_{\mathrm{B}} T / \hbar, \omega$ is the frequency of incident light, $e$ is the charge of an electron, $\tau$ is the Drude relaxation rate, $T$ is the temperature, and $k_{\mathrm{B}}$ is the Boltzmann constant. The first term in eq 1 describes the free-carrier (intraband) response of graphene. The second term captures the response due to the interband transitions. We should also note that the above expression of $\sigma(\omega)$ assumes no spatial dispersion and is also referred to as local limit $\left(k_{\|} \rightarrow 0\right)$ of RPA in the literature.

Figure $1 \mathrm{~b}$ shows the dimensions of the fabricated plasmonic antennas that were optimized to achieve a resonance at a $2 \mu \mathrm{m}$ wavelength using full-wave 3D finite element (FE) frequency domain simulations (with COMSOL Multiphysics). The antennas were fabricated on a graphene FET by e-beam lithography using $100 \mathrm{~nm}$ ZEP 520A (Zeon Chemicals) as a resist. The exposure doses of individual elements in the unit cell were optimized to achieve reliable gaps of 20 and $50 \mathrm{~nm}$. This was followed by e-beam evaporation of $2 \mathrm{~nm} \mathrm{Ti} / 20 \mathrm{~nm} \mathrm{Au}$ and subsequent lift-off processes. The SEM micrograph in Figure 3c shows a represtative dimensions of the fabricated sample. The optical studies of the samples were performed using a Fourier transform infrared spectrometer (FTIR) in the reflection mode.

To verify the hypothesis that Fano resonant structures interact strongly with SLG, we measured the reflectance from the antennas at four different locations with and without an underlying SLG as shown in Figure 2. We observe a strong impact of the graphene on the spectra measured and also the characteristic the Fano dip in line shape around $1.8 \mu \mathrm{m}$. Further, the spectra measured from the antennas fabricated on the graphene at different locations show small deviations, while spectra from antennas fabricated on bare substrate are identical. This can be easily explained by the spatial inhomogeneities of

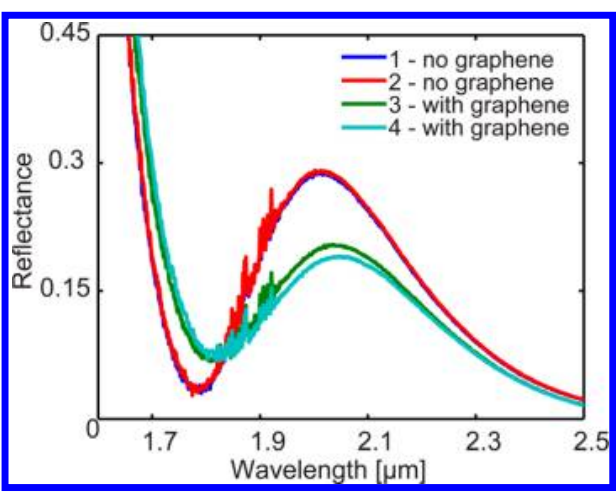

Figure 2. Measured optical reflectance spectra from Fano resonant antennas at four different locations (two without graphene and two with graphene) showing strong impact of graphene on Fano resonance. In measurements without graphene (red and blue curves), there is perfect overlap, while measurement with graphene (green and turquoise curves) show variations due to spatial inhomogeneties. The dip at $1.8 \mu \mathrm{m}$ corresponds to location of the Fano resonance (overlapping dipolar and quadupolar modes in dolmen geometry). We note that in this particular sample the reflection at lower wavelengths doesnot decrease as expected in Fano line shape due to fabrication imperfections. 
carrier density due to topographic corrugations ${ }^{46}$ and electron and hole puddles ${ }^{47}$ that lead to changes in the optical properties. It should be noted that while this strong impact of graphene on the Fano resonance agrees with other studies, $^{26,48,49}$ it is only the first step in the realization of a practical device with large dynamic tunability.

A large doping induced change in dielectric function of graphene is essential to achieve strong tunability at visible and near-IR frequencies. This can be experimentally realized when carrier densities around $1 \times 10^{13} \mathrm{~cm}^{-2}$ are reached. ${ }^{32}$ Such high carrier densities are very difficult to achieve through the conventional back-gate doping using a $300 \mathrm{~nm}$ oxide. ${ }^{50}$ Instead we used electrochemical gating with DEME-TFSI (Diethylmethyl (2-methoxyethyl) ammonium bis (trifluoromethylsulfonyl) imide) as a top electrolyte to achieve the required large doping of graphene. Ionic liquid electrolytes have a large capacitance of $\sim 1-10 \mu \mathrm{Fcm}^{-2}$ because of the formation of extremely thin electric double layers (EDL) on the surface of graphene channel. ${ }^{51}$ This EDL helps in achieving a strong capacitive control on the channel making it possible to realize a large carrier injection. For a particular applied voltage, the carrier density in ion-gel gated graphene is higher by orders of magnitude compared to back gating using $\mathrm{SiO}_{2}$ gate dielectrics (see the carrier density estimatation in SOM). Figure 3 shows measurements performed using the same device via back gating and ion-gel gating. We clearly see that introduction of the ion-gel causes the resonance to be redshifted. At the same time, the tunability of the resonance

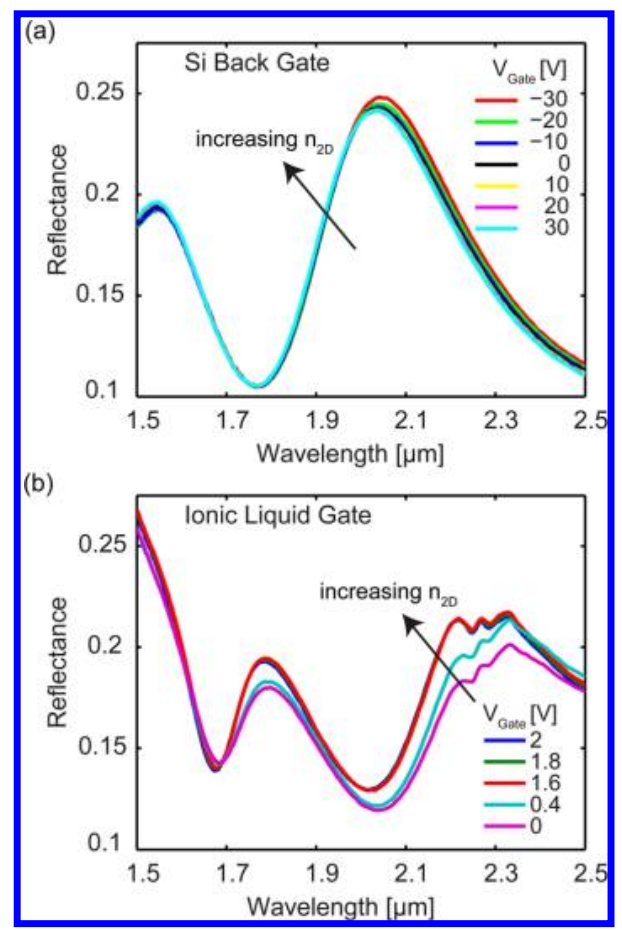

Figure 3. (a) Experimentally measured modulation of the resonance using backgating through $\mathrm{SiO}_{2}$. The measurements were performed in hole doping regime where carrier concentration was reduced with the applied voltage increase. (b) Experimentally measured optical spectra on the same device using ion-gel top electrolyte gating. The scans were taken in the electron doping regime where carrier concentration increases as the applied voltage increases. See Supporting Information Figure $S 1 \mathrm{a}, \mathrm{b}$ for the changes in source-drain resistance $\left(R_{\mathrm{SD}}\right)$ with gate voltage $\left(V_{\mathrm{G}}\right)$ measured during optical measurements. increases considerably. Figure 4a shows optical mesurements using ion-gel gating measured on one of the fabricated devices which shows a similar trend as in Figure $3 b$.

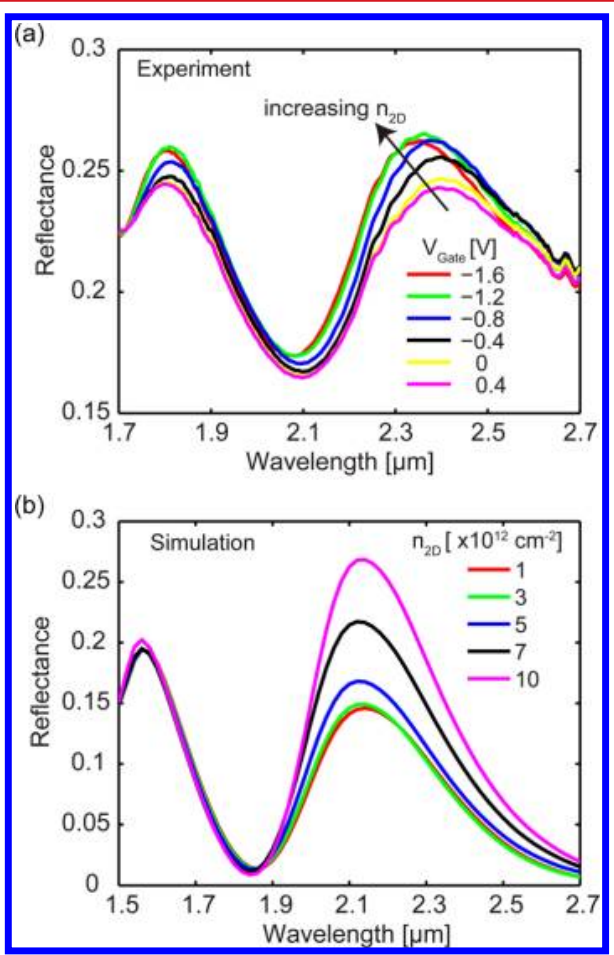

Figure 4. (a) The measured optical reflectance spectra with ion-gel top electrolyte. See Supporting Information Figure S1c for the variation in source-drain resistance $\left(R_{\mathrm{SD}}\right)$ with gate voltage $\left(V_{\mathrm{G}}\right)$ measured during optical measurement. (b) Three-dimensional FE simulations using experimental geometry and graphene sheet carrier density as an input parameter. We observe a qualitative agreement with experimental results. These simulations do not take into account the ion-gel whose thickness and index are challenging to determine in the experiment. This explains the difference in the experimentally measured and simulated wavelength of the Fano dip.

To verify the experimental observations we performed numerical simulations using 3D FE modeling of SLG as a transition boundary condition wherein the surface current depends on the conductivity given by eq 1 . The simulation results which are shown in Figure $4 \mathrm{~b}$ show qualitative agreement with experimental results. We note that the peak to the left of Fano dip shows less modulation than the peak to the right of Fano dip. This is consistent with our previous results $^{32}$ showing the impact of graphene to be stronger at longer wavelengths. Further, the measured data show a saturation effect, wherein the spectra do not significantly change at large carrier concentrations. This differs from our simulation results that assume an ideal scenario of increasing carrier concentration. This clearly indicates that the graphene carrier concentration around the gold antennas shows a much smaller degree of variation than the changes expected from freestanding graphene. This can result from the work function mismatch leading to contact resistance between the graphene and gold nanostructures. ${ }^{52}$ This can be minimized by introducing thin intermediate metals and is beyond the scope of this work. Another potential direction for improving the tunability of the plasmonic resonance is using several layers of 
graphene which have higher optical conductivity, ${ }^{53}$ therefore leading to stronger impact on plasmonic resonance.

Because the quadrupolar mode in a plasmonic Fano structure does not couple directly to radiation, its primary decay path is the generation of e-h pairs leading to efficient generation of hot electrons. Hot electrons generated in plasmonic Fano resonances at visible wavelengths have been shown to contribute to doping of graphene. ${ }^{49}$ In our work, we do not observe this light induced doping effect possibly due to the fact that the incident FTIR beam is quite weak and we do not have sufficient number of hot electrons to see a measurable effect.

In summary, we have demonstrated efficient dynamic control of Fano resonances in plasmonic structures at NIR wavelengths using graphene. Stronger modulation can be achieved by optimizing the graphene nanostructure contact resistance and by use of multilayer graphene. This demonstration paves the way for development of tunable elements for next generation of plasmonic and hybrid nanophotonic on-chip devices such as sensors and detectors. In the future, alternative approaches like optical pumping of electrons ${ }^{54}$ into conduction band can help in improving the modulation speed and enable of ultrafast devices.

\section{ASSOCIATED CONTENT}

\section{S Supporting Information}

Additional information regarding samples and $V_{\mathrm{G}}-R_{\mathrm{SD}}$ characteristics of samples used in Figures 3 and 4 is provided. This material is available free of charge via the Internet at http://pubs.acs.org.

\section{AUTHOR INFORMATION}

\section{Corresponding Author}

*E-mail: aeb@purdue.edu.

\section{Notes}

The authors declare no competing financial interest.

\section{ACKNOWLEDGMENTS}

This work was supported in part by ARO MURI Grant 56154PH-MUR (W911NF-09-1-0539) and ARO Grant 57566PHRIP (W911NF-10-1-0380). A.V.K. was supported in part by AFRL Materials and Manufacturing Directorate Applied Metamaterials Program. The graphene synthesis and FET device fabrication were supported in part by NSF (DMR 0847638) and DTRA (HDTRA1-09-1-0047). We also thank Professor Oana Malis for allowing the use of FTIR in her lab.

\section{REFERENCES}

(1) Lal, S.; Link, S.; Halas, N. J. Nat. Photonics 2007, 1 (11), 641648.

(2) Brongersma, M. L.; Shalaev, V. M. Science 2010, 328 (5977), $440-441$.

(3) Gramotnev, D. K.; Bozhevolnyi, S. I. Nat. Photonics 2010, 4 (2), $83-91$.

(4) Atwater, H. A. Sci. Am. 2007, 296 (4), 56-62.

(5) Stipe, B. C.; Strand, T. C.; Poon, C. C.; Balamane, H.; Boone, T. D.; Katine, J. A.; Li, J.-L.; Rawat, V.; Nemoto, H.; Hirotsune, A. Nat. Photonics 2010, 4 (7), 484-488.

(6) Huang, X.; Jain, P. K.; El-Sayed, I. H.; El-Sayed, M. A. Lasers Med. Sci. 2008, 23 (3), 217-228.

(7) Anker, J. N.; Hall, W. P.; Lyandres, O.; Shah, N. C.; Zhao, J.; Van Duyne, R. P. Nat. Mater. 2008, 7 (6), 442-453.

(8) Endo, T.; Kerman, K.; Nagatani, N.; Hiepa, H. M.; Kim, D.-K.; Yonezawa, Y.; Nakano, K.; Tamiya, E. Anal. Chem. 2006, 78 (18), 6465-6475.
(9) Chen, H. T.; Padilla, W. J.; Zide, J. M. O.; Gossard, A. C.; Taylor, A. J.; Averitt, R. D. Nature 2006, 444 (7119), 597-600.

(10) Chen, H.-T.; O'Hara, J. F.; Azad, A. K.; Taylor, A. J.; Averitt, R. D.; Shrekenhamer, D. B.; Padilla, W. J. Nat. Photonics 2008, 2 (5), 295-298.

(11) Fu, Y. H.; Liu, A. Q.; Zhu, W. M.; Zhang, X. M.; Tsai, D. P.; Zhang, J. B.; Mei, T.; Tao, J. F.; Guo, H. C.; Zhang, X. H. Adv. Funct. Mater. 2011, 21 (18), 3589-3594.

(12) Ou, J.-Y.; Plum, E.; Zhang, J.; Zheludev, N. I. Nat. Nanotechnol. 2013, 8 (4), 252-255.

(13) Xiao, S.; Chettiar, U. K.; Kildishev, A. V.; Drachev, V.; Khoo, I.; Shalaev, V. M. Appl. Phys. Lett. 2009, 95 (3), 033115-033115-3.

(14) Feigenbaum, E.; Diest, K.; Atwater, H. A. Nano Lett. 2010, 10 (6), 2111-2116.

(15) Naik, G. V.; Shalaev, V. M.; Boltasseva, A. Adv. Mater. 2013, 25 (24), 3264-3294.

(16) Maier, S. A., Plasmonics: Fundamentals and Applications; Springer: New York, 2007.

(17) Wang, F.; Zhang, Y.; Tian, C.; Girit, C.; Zettl, A.; Crommie, M.; Shen, Y. R. Science 2008, 320 (5873), 206-209.

(18) Li, Z.; Henriksen, E.; Jiang, Z.; Hao, Z.; Martin, M.; Kim, P.; Stormer, H.; Basov, D. N. Nat. Phys. 2008, 4 (7), 532-535.

(19) Fang, Z.; Thongrattanasiri, S.; Schlather, A.; Liu, Z.; Ma, L.; Wang, Y.; Ajayan, P. M.; Nordlander, P.; Halas, N. J.; García de Abajo, F. J. ACS Nano 2013, 7 (3), 2388-2395.

(20) Chen, J.; Badioli, M.; Alonso-Gonzalez, P.; Thongrattanasiri, S.; Huth, F.; Osmond, J.; Spasenovic, M.; Centeno, A.; Pesquera, A.; Godignon, P.; Zurutuza Elorza, A.; Camara, N.; Javier Garcia de Abajo, F.; Hillenbrand, R.; Koppens, F. H. L. Nature 2012, 487 (7405), 7781

(21) Fei, Z.; Rodin, A. S.; Andreev, G. O.; Bao, W.; McLeod, A. S.; Wagner, M.; Zhang, L. M.; Zhao, Z.; Thiemens, M.; Dominguez, G.; Fogler, M. M.; Neto, A. H. C.; Lau, C. N.; Keilmann, F.; Basov, D. N. Nature 2012, 487 (7405), 82-85.

(22) Brar, V. W.; Jang, M.; Sherrott, M.; Lopez, J. J.; Atwater, H. A. Nano Lett. 2013, 13 (6), 2541-2547.

(23) Yan, H.; Li, X.; Chandra, B.; Tulevski, G.; Wu, Y.; Freitag, M.; Zhu, W.; Avouris, P.; Xia, F. Nat. Nanotechnol. 2012, 7 (5), 330-334.

(24) Ju, L.; Geng, B. S.; Horng, J.; Girit, C.; Martin, M.; Hao, Z.; Bechtel, H. A.; Liang, X. G.; Zettl, A.; Shen, Y. R.; Wang, F. Nat. Nanotechnol. 2011, 6 (10), 630-634.

(25) Papasimakis, N.; Thongrattanasiri, S.; Zheludev, N. I.; de Abajo, F. G. Light: Sci. Appl. 2013, 2 (7), e78.

(26) Papasimakis, N.; Luo, Z.; Shen, Z. X.; De Angelis, F.; Di Fabrizio, E.; Nikolaenko, A. E.; Zheludev, N. I. Opt. Express 2010, 18 (8), 8353-8359.

(27) Liu, M.; Yin, X.; Ulin-Avila, E.; Geng, B.; Zentgraf, T.; Ju, L.; Wang, F.; Zhang, X. Nature 2011, 474 (7349), 64-67.

(28) Fang, Z.; Liu, Z.; Wang, Y.; Ajayan, P. M.; Nordlander, P.; Halas, N. J. Nano Lett. 2012, 12 (7), 3808-3813.

(29) Freitag, M.; Low, T.; Zhu, W.; Yan, H.; Xia, F.; Avouris, P. Nat. Commun. 2013, 4.

(30) Vakil, A.; Engheta, N. Science 2011, 332 (6035), 1291-1294.

(31) García de Abajo, F. J. Science 2013, 339 (6122), 917-918.

(32) Emani, N. K.; Chung, T.-F.; Ni, X.; Kildishev, A. V.; Chen, Y. P.; Boltasseva, A. Nano Lett. 2012, 12 (10), 5202-5206.

(33) Yao, Y.; Kats, M. A.; Genevet, P.; Yu, N.; Song, Y.; Kong, J.; Capasso, F. Nano Lett. 2013, 13 (3), 1257-1264.

(34) Li, Z.; Yu, N. Appl. Phys. Lett. 2013, 102 (13), 131108$131108-5$.

(35) Kim, J.; Son, H.; Cho, D. J.; Geng, B.; Regan, W.; Shi, S.; Kim, K.; Zettl, A.; Shen, Y.-R.; Wang, F. Nano Lett. 2012, 12 (11), 55985602.

(36) Vasic, B.; Jakovljevic, M. M.; Isic, G.; Gajic, R. Appl. Phys. Lett. 2013, 103 (1), 011102-011102-4.

(37) Majumdar, A.; Kim, J.; Vuckovic, J.; Wang, F. Nano Lett. 2013, 13 (2), 515-518.

(38) Shankar, R.; Yao, Y.; Frish, J.; Frank, I.; Song, Y.; Kong, J.; Capasso, F.; Loncar, M. In Electro-Optic Tuning of Mid-infrared 
Photonic Crystal Cavities using Graphene, CLEO: Science and Innovations, San Jose, June 9, 2013; Optical Society of America: San Jose, 2013; p CTu1F.5.

(39) Luk'yanchuk, B.; Zheludev, N. I.; Maier, S. A.; Halas, N. J.; Nordlander, P.; Giessen, H.; Chong, C. T. Nat. Mater. 2010, 9 (9), $707-715$.

(40) Verellen, N.; Sonnefraud, Y.; Sobhani, H.; Hao, F.; Moshchalkov, V. V.; Dorpe, P. V.; Nordlander, P.; Maier, S. A. Nano Lett. 2009, 9 (4), 1663-1667.

(41) Dregely, D.; Hentschel, M.; Giessen, H. ACS nano 2011, 5 (10), $8202-8211$.

(42) Cao, H.; Yu, Q.; Jauregui, L. A.; Tian, J.; Wu, W.; Liu, Z.; Jalilian, R.; Benjamin, D. K.; Jiang, Z.; Bao, J. Appl. Phys. Lett. 2010, 96, 122106.

(43) Chung, T. F.; Shen, T.; Cao, H.; Jauregui, L. A.; Wu, W.; Yu, Q.; Newell, D.; Chen, Y. P. Int. J. Mod. Phys. B 2013, 27, 10.

(44) Falkovsky, L. In Optical properties of graphene; IOP Publishing: Washington, DC, 2008, p 012004.

(45) Koppens, F. H. L.; Chang, D. E.; García de Abajo, F. J. Nano Lett. 2011, 11 (8), 3370-3377.

(46) Zhang, Y.; Brar, V. W.; Girit, C.; Zettl, A.; Crommie, M. F. Nat. Phys. 2009, 5 (10), 722-726.

(47) Martin, J.; Akerman, N.; Ulbricht, G.; Lohmann, T.; Smet, J.; Von Klitzing, K.; Yacoby, A. Nat. Phys. 2007, 4 (2), 144-148.

(48) Mousavi, S. H.; Kholmanov, I.; Alici, K. B.; Purtseladze, D.; Arju, N.; Tatar, K.; Fozdar, D. Y.; Suk, J. W.; Hao, Y.; Khanikaev, A. B. Nano Lett. 2013, 13 (3), 1111-1117.

(49) Fang, Z.; Wang, Y.; Liu, Z.; Schlather, A.; Ajayan, P. M.; Koppens, F. H.; Nordlander, P.; Halas, N. J. ACS Nano 2012, 6 (11), $10222-10228$.

(50) Das, A.; Pisana, S.; Chakraborty, B.; Piscanec, S.; Saha, S.; Waghmare, U.; Novoselov, K.; Krishnamurthy, H.; Geim, A.; Ferrari, A.; Sood, A. Nat. Nanotechnol. 2008, 3 (4), 210-215.

(51) Fujimoto, T.; Awaga, K. Phys. Chem. Chem. Phys. 2013, 15 (23), 8983-9006.

(52) Lee, E. J.; Balasubramanian, K.; Weitz, R. T.; Burghard, M.; Kern, K. Nat. Nanotechnol. 2008, 3 (8), 486-490.

(53) Mak, K. F.; Sfeir, M. Y.; Misewich, J. A.; Heinz, T. F. Proc. Natl. Acad. Sci. 2010, 107 (34), 14999-15004.

(54) Dubinov, A.; Aleshkin, V. Y.; Mitin, V.; Otsuji, T.; Ryzhii, V. J. Phys.: Condens. Matter 2011, 23 (14), 145302. 